\title{
Staining of bone marrow aspiration slides: novel immediate staining Vs EDTA-stored samples
}

\begin{abstract}
Background: Bone Marrow Biopsy is used as an intervention to diagnose certain hematological and systemic diseases as an adjunct to routine laboratory investigations. The procedure includes getting an aspirate and a trephine biopsy. Slides/Smears are prepared from the aspirate and touch imprints along with Hematoxylin and Eosin $(\mathrm{H}$ and E) stained sections are prepared from the trephine. Traditionally the slides from the aspirate have been prepared directly (without anticoagulants) and examined along with the trephine biopsy sections to reach a diagnosis. EDTA (Ethylene Diammine Tetra Acetate) preserved specimen can also be used to make slides of the aspirate.
\end{abstract}

Objective: To compare two methods of bone marrow aspirate preparation.

Design of study: Randomized controlled trial.

Place of study: Department of Pathology, Pakistan Institute of Medical Sciences, Islamabad

Materials and methods: Patients coming to the Department of Pathology for bone marrow biopsy had their samples taken. Half of each sample was used to make direct smears and the other half was preserved in EDTA i.e. the purple top vials. Slides were made at the end of the procedure by the preserved sample and then the two were stained by the same person and procedure (Wright stain) and examined for any differences in quality. SPSS version 21.2 was used to analyze the data.

Results: A total of 132 was taken.77 (58.3\%) were males and 55(41.7\%) were females. $50(37.9 \%)$ were adults and $82(62.1 \%)$ were children. $\mathrm{P}-\mathrm{value}$ was found to be 0.81392 which was non-significant proving the fact that the 2 techniques are comparable.

Conclusion: EDTA preserved bone marrow aspirate can be used to prepare slides at the end of the whole procedure without compromising the quality of the smears and result interpretation.

Keywords: bone marrow aspirate, EDTA, aspirate smears, trephine biopsy
Volume 6 Issue 6 - 2018

\author{
Asfa Zawar,' Shahzad Ali Jiskani,' Maryam \\ Zulfiqar,' Aliena Sohail,' Asma Mustafa,' \\ Bushra Anam Ali,' Sundas Ali, ${ }^{2}$ Sarah Jamal ${ }^{2}$ \\ 'Pakistan Institute of Medical Sciences, Pakistan \\ ${ }^{2}$ Pakistan Atomic Energy Commission General Hospital, Pakistan
}

Correspondence: Shahzad Ali Jiskani, Shaheed Zulfiqar Ali Bhutto Medical University/ Pakistan Institute of Medical Sciences Islamabad, House No. 482, Behrani Muhallah, Tando Muhammad Khan, Pakistan, Tel 3332672268, Email shahzadbalooch289@gmail.com

Received: September 29, 2018 | Published: November 15, 2018

\section{Introduction}

Bone marrow tissue provides the necessary environment for the production and development of the blood cell precursors. The bone marrow has a network of sinusoids which are lined by the endothelial cells. Along with these the blood cell precursors and the supporting tissue like the fat cells and the extracellular matrix are located outside the vessels. ${ }^{1}$

Bone marrow aspiration and biopsy is a necessary procedure needed for the diagnosis and monitoring the course of various hematological disorders like leukemias, plasma cell disorders, bone marrow failure syndromes, congenital dys-erythropoietic syndromes and other non hematological disorders like pyrexia of unknown origin, leishmaniasis and infection in the body. Sample can be obtained for bone marrow culture and immunophenotyping in case of leukemias which can yield useful information. ${ }^{1-4}$

Fibrosis can be seen and its degree assessed on the trephine biopsy sections. ${ }^{5}$ Obtaining the desirable quality specimen is obviously important for reporting such an invasive procedure. ${ }^{2}$ It is important before performing the biopsy to see if it is clinically indicated. Then comes the proper aspirate collection, preparation and staining of smears and in the end the systematic review of the sample. This needs adequate knowledge of the normal architecture of bone and proportion of all cell lines in the bone marrow. Current complete blood picture along with the bone marrow aspirate and trephine biopsy sections is required to reach a diagnosis and these two complement each other. ${ }^{6,7}$

Bone marrow aspirate and biopsy is performed usually from the posterior superior iliac spine in adults and children above 2years of age under local anesthesia. Other sites include the anterior superior iliac spine, the tibia (especially in children under 2years of age), spinous processes of the vertebrae and the sternum. Satisfactory bone marrow aspirate sample which has adequate bone fragments is needed for proper reporting. Generally this is the first aspirated sample. ${ }^{8}$

International Committee for Standardization in Hematology (ICSH) recommends the use of two types preparations for examination under the microscope: Wedge-spread film and crush film/ touch preparations/ imprints. Wedge spread films are used to assess the cellularity of the bone marrow, morphology of all cells and any other special or different feature in the cell lines. Crush film is considered better because the aspirate can be diluted with blood during collection and also the neoplastic cells might have a focal growth pattern. ${ }^{1}$

Problems related to the bone marrow aspirate and its interpretation includes: 
I. Performing an aspirate that was not necessary

II. Difficulty in performing a clinically indicated aspirate

III. Diluted aspirate/Blood mixed tap

IV. Misinterpretation of the findings

V. Inexpert performer

VI. Inappropriate handling of the specimen

If the performer is aware of the pitfalls and is adequately trained, he can reduce the errors in bone marrow reporting. ${ }^{9}$

Since the very beginning, smears are prepared directly using non-anticoagulated aspirate. EDTA preserved specimen can also be used to make the slides. There are not many studies comparing the effectiveness of these two methods of smear preparation. ${ }^{10}$

The traditional method of preparing direct smears can help reduce some of the post- collection errors such as dilution due to excess anticoagulant and storage artifacts. However if the performer makes haste in preparing slides using the non-anticoagulant preserved sample, he may produce poor quality smears. Anticoagulant decreased the risk of clotting of the sample and thus gives the person preparing the slides enough time to make good quality slides. ${ }^{8}$

\section{Materials and methods}

This is a cross sectional comparative study carried out at the Department of Pathology Pakistan Institute of Medical Sciences Islamabad, Pakistan. The total duration was 3 months from January 2018 to March 2018.The sampling technique used was nonprobability consecutive sampling. All the patients coming for the bone marrow biopsy were included in the study. The dry and diluted taps were excluded as were those that came just for cytogenetics/ immunophenotyping.

The patients provided informed consent. Brief medical history was taken from all the patients. All the relevant laboratory reports were noted. Local anesthesia was given and bone marrow biopsy was performed. The site of biopsy for the adults and children aged above 2 years was posterior superior iliac spine and the anterior iliac spine, only in cases where the patient was obese or had a bed sore or surgical wound preventing them from making the right position for the aspirate to be taken from the posterior iliac spine. 0.5 to $1 \mathrm{~mL}$ of bone marrow aspirate was taken; half quantity was used to make direct smears and the other half was saved in the purple top vial containing EDTA as the anticoagulant. Smears from the anticoagulant mixed aspirate were made at the end of the procedure (5-30 minutes window period). The slides were stained and observed for the quality, fragments, stain, readability and results will be compared. Preparation of smears and staining was done by the same person/expert to avoid errors based on change in the performer. A time more than 30 minutes was not given.

Adults were the patients with ages above 12 years. 12years and below were considered as children.

\section{Outcome}

Outcome were measured in terms of score based on the following parameters

\section{Quality of smear}

Based on the length, width, head, body and tail of the slide the quality will be judged ( $4=$ Excellent, $3=$ Good, $2=$ Average, $1=$ Poor, $0=$ Worst).

\section{Fragments}

Presence and absence of fragments ( $1=$ Present, $0=$ Absent $)$

\section{Staining}

Wright stain was used to stain the slides and then comparison was made on the basis of quality of staining ( $4=$ Excellent, $3=$ Good, $2=$ Average, $1=$ Poor, $0=$ Worst).

\section{Readability}

Reproducibility of the results after examination of the slides of both types of smears was seen whether it's same or different (4=Excellent, $3=$ Good, $2=$ Average, $1=$ Poor, $0=$ Worst).

The data was analyzed using SPSS version 21.0. P-value of $<0.05$ was considered as statistically significant.

\section{Results}

A total of 141 cases were taken out of which one of the patients refused the procedure. 2 out of these 140 were diluted aspirates and 6 were samples taken for cytogenetics/ immunophenotyping so these 8 were not included according to the scoring scale made. This reduced the sample size to $132(\mathrm{n}=132)$. Out of the 132 patients included in the study, $77(58.3 \%)$ were males and $55(41.7 \%)$ were females (Figure 1). $50(37.9 \%)$ patients were adults and $82(62.1 \%)$ were children (Figure 2) All cases were scored according to quality (Figure 3). Cases stained with routine immediate staining showed $109(82.57 \%)$ as excellent quality (score 4), $17(12.87 \%$ ) as good quality (score 3$), 5(3.78 \%)$ as average quality (score 2 ) and $1(0.75 \%)$ as poor quality (score 1$)$. No case received score 0. In samples stored with EDTA, 106 (80.30\%) were of excellent quality (score 4$), 19(14.39 \%)$ were good quality (score 3), 5 (3.78\%) were average quality (score 2) and $2(1.51 \%)$ were poor quality. There was also no case with score 1 . There was no statistical significant difference in both techniques $(\mathrm{p}=0.813) .{ }^{11-13}$

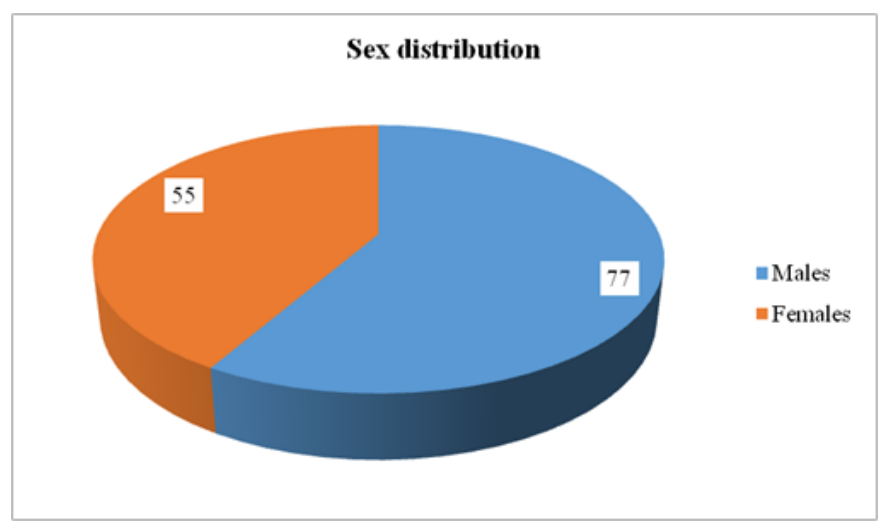

Figure I Gender Distribution $(n=\mid 32)$. 


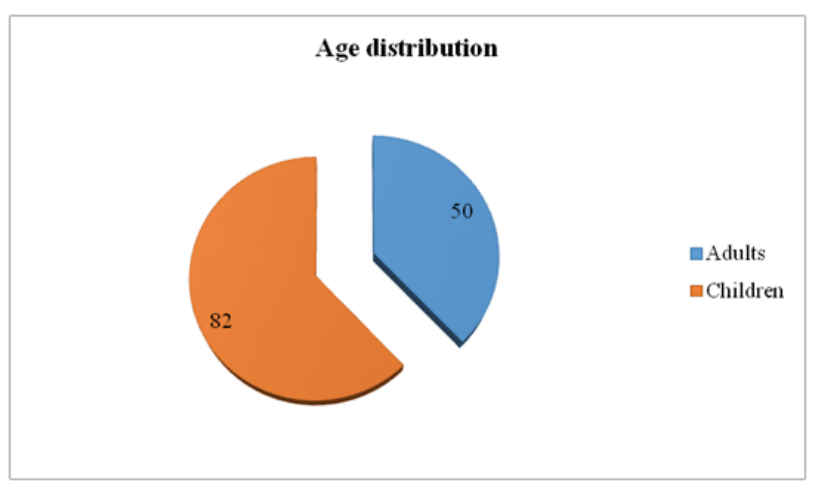

Figure 2 Age Distribution $(n=\mid 32)$.

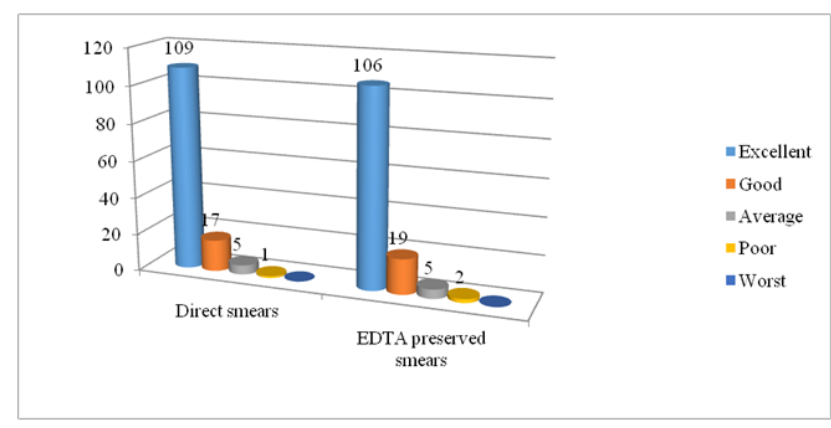

Figure 3 Number of patients according to scores.

\section{Discussion}

Bone marrow biopsy is a procedure that we need for the diagnosis of certain diseases and for monitoring the response to treatment and progression of disease when on treatment in others like leukemias, plasma cell disorders, bone marrow failure syndromes, congenital dys-erythropoietic syndromes and other non hematological disorders like pyrexia of unknown origin, leishmaniasis and infection in the body. Sample can be obtained for bone marrow culture and immunophenotyping in case of leukemias which can yield useful information. ${ }^{1-4}$ Sites for aspiration and biopsy include posterior superior iliac spine, the anterior iliac spine, sternum and the vertebral spinous processes in case of adults and anterior tibia in case of children below 2 years of age. Satisfactory bone marrow aspirate sample which has adequate bone fragments is needed for proper reporting. Generally this is the first aspirated sample. ${ }^{8}$

Aspirates are used to make smears which help in assessing the cellularity of the marrow and the morphology of the cells. Touch preparations or imprints are also prepared using the trephine biopsy specimen and are considered better because the aspirate can be diluted with blood during collection and also the neoplastic cells might have a focal growth pattern. ${ }^{1}$

Usually the smears are prepared using the non-anticoagulated specimen of the bone marrow aspirate EDTA preserved specimen can also be used to make the slides. There are not much studies comparing the effectiveness of these two methods of smear preparation. ${ }^{10}$

Aleem et al. ${ }^{8}$ has carried out a similar study in Saudi Arabia at King Khalid Hospital where they have worked on a total 238 samples and collected this in a period of almost 3years. Their results were statistically non significant too proving that the two techniques are similar and EDTA preserved smears can be utilized for result interpretation. ${ }^{8}$ Di Francesco et al..$^{10}$ has demonstrated a similar result in which the preparation of the slides in the laboratory by anticoagulated blood which was collected at the patient's bedside while carrying out the biopsy procedure provided good results. They used 129 slides and they were interpreted by 3 readers. ${ }^{10}$

\section{Conclusion}

The two techniques of bone marrow aspirate smear preparation are similar. The quality of the slides prepared by the preserved smear is not significantly different from the direct smears. Thus this method of preserving the aspirate in EDTA can be used especially when the technician preparing them needs time assisting the trephine biopsy procedure or lack of expertise. The slides can be prepared at the end of the procedure in an easy and relaxed manner without compromising the quality of the aspirate and its interpretation. This EDTA preservation can also be helpful if small amount of aspirate has been obtained and there is a chance of it to clot in the syringe before making the slides. Not many studies have been seen when EDTA preservation of the bone marrow is considered. We have provided a maximum of 30 minutes time for using the preserved aspirate. There is a chance that if the aspirate is kept for a longer duration the storage artifacts appears. This can be proved by further studies.

\section{Acknowledgements}

None.

\section{Conflict of interest}

Authors declare that there is no conflict of interest.

\section{References}

1. Lewandowski K, Complak A, Hellmann A. Microscopic examination of bone marrow aspirates in malignant disorders of haematopoiesis-a comparison of two slide preparation techniques. Ann Hematol. 2012;91(4):497-505.

2. Grønkjær M, Hasselgren CF, Østergaard AS, et al. Bone Marrow Aspiration: A Randomized Controlled Trial Assessing the Quality of Bone Marrow Specimens Using Slow and Rapid Aspiration Techniques and Evaluating Pain Intensity. Acta Haematol. 2016;135(2):81-87.

3. Temiz F, Gürbüz BB, Leblebisatan G, et al. An Association of Leishmaniasis and Dyserythropoiesis in Children. Indian J Hematol Blood Transfus. 2014;30(1):19-21.

4. Sheikh NS, Sheikh AS, Hussain SI, et al. Utility of thick smears of bone marrow aspirate in pyrexia of unknown origin. $J$ Coll Physicians Surg Pak. 2003;13(10):577-580.

5. Tefferi A. Primary myelofibrosis_2017 update on diagnosis, riskstratification, and management. Am J Hematol. 2016;91(12):1262-1271.

6. Brynes RK, McKenna RW, Sundberg RD. Bone marrow aspiration and trephine biopsy. Am J Clin Pathol. 1978;70(5):753-759.

7. Stacy NI, Harvey JW. Bone Marrow Aspirate Evaluation. Vet Clin North Am Small Anim Pract. 2017;47(1):31-52.

8. Aleem A, Alsaleh K, Aljabry M, et al. A comparison of two techniques of preparing bone marrow aspirate slides. J Pak Med Assoc. 2016;66(5):528-527.

9. Bain BJ, Bailey K. Pitfalls in obtaining and interpreting bone marrow aspirates- to err is human. J Clin Pathol. 2011;64(5):373-379. 
10. DiFrancesco T, Boychuk DJ, Lafferty JD, et al. Bone marrow aspirate collection and preparation-a comparison of three methods. Clin Invest Med. 2012;35(3):E114-6.

11. Dorji K, Tobgay T, Jamtsho R, et al. Microscopic Image of LeishmanDonovan Bodies in Bone Marrow Aspirate Smear of Patient Suffering from Unexplained Intermittent Low-Grade Fever and Cough. Turk $J$ Haematol. 2017;34(3):266-267.
12. Bain B, Bates I, Laffan M, et al. Dacie and Lewis practical haematology. $11^{\text {th }}$ ed. UK: Churchill Livingstone; 2011. p. 668.

13. Bain BJ. Bone Marrow Aspiration. J Clin Pathol. 2001;54(9):657-663. 